\title{
Transtibial vs Anatomical tunneling techniques for arthroscopic ACL Reconstruction in non-athletic population
}

\author{
Ali Electricwala ${ }^{1}$, Chintamani Latkar ${ }^{1}$, Sanjay Patil ${ }^{1}$, Vilas Jog ${ }^{1}$, Amit Mahajan ${ }^{1}$ \\ Shantanu Deshpande
}

${ }^{1}$ Department of Orthopaedics, BharatiVidyapeeth University, Pune, India

Institute at which research was conducted: Bharati Vidyapeeth University, Pune, India.

University Affiliation of Thesis: BharatiVidyapeeth University, Pune, India. Year of Acceptance: 2013

Address of Correspondence

Dr Ali Electricwala.

Department of Orthopaedics, BharatiVidyapeeth University, Pune, India E mail: ali.electricwala@gmail.com
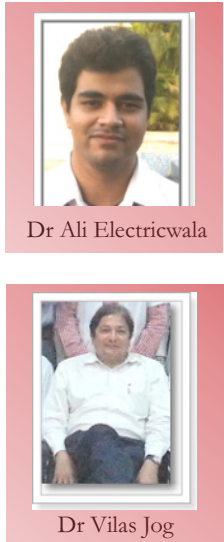
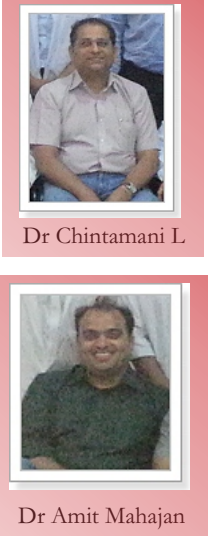
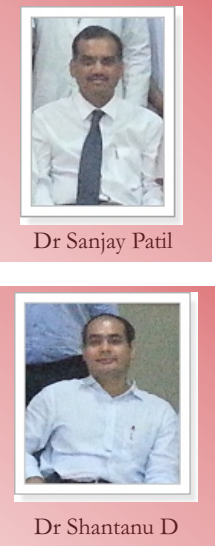

Abstract: Background: Transtibial tunneling technique has been the gold standard for arthroscopic ACL reconstruction for many years,. Despite this high level of success, a growing body of literature has questioned whether this technique sufficiently recreates the anatomy and function of the native ACL. This created a vogue amongst the arthoscopists for anatomical ACL reconstruction using the anteromedial portal. The purpose of this study was to compare the stability and functional outcome using both the techniques.

Materials and methods: 50 patients (39males and 11 females),all non-athletes with ACL deficient knees underwent ACL reconstruction, 25 by transtibial and 25 by anatomical technique. on the basis on stability using Lachman's and Slocum's tests and functional outcome using Lysholm knee score at 3,6 and 12 months.

Result: There was no significant difference in the Functional outcome (Lysholm Knee score), anteroposterior stability (Lachman's test) and rotational stability (Slocum's test) \{p values $>0.05\}$.

Conclusion: Both groups have equally good stability in both the anteroposterior and rotational plane.

Keywords: Transtibial tunnel, anatomical tunnel, non athelete, ACL injury

\section{THESIS SUMMARY}

\section{Introduction:}

Anterior Cruciate ligament (ACL) injuries of the knee are verycommantoday due increasing incidence of road traffic accidents and sports injuries. ACL autograft can be prepare dusing Bone-Tendon-Bone (BTB) or Hamstring graft harvest. For ACL reconstuction, tibial tunnel is prepared using a standard jig.The femoral tunnel can be prepared either through the tibial tunnel(Transtibial) or through the anteromedial portal(Anatomical).Transtibial tunneling technique has been the gold standard for arthroscopic ACL reconstruction positioned at ideal 10 or 2 o clock positions for many years. Despite this high level of ensuring better rotational stability. The success, a growing body of literature has advantages of transtibial technique are questioned whether this technique sufficiently straight guide wire tunnel ,technically easy, re-creates the anatomy and function of the Longer and less oblique tunnel giving better native ACL. The advantage of anteromedial AP stability and endobutton is resting on good portal are femoral and tibial tunnels are drilled cortical bone. The disadvantages of independently of each other,allows anteromedial portal are shorter tunnel hence preservation of any remaining intact ACL less AP stability ,risk of peroneal nerve injury fibers, allowing isolated reconstruction of the ,femoral tunnel must be drilled with the knee anteromedial or posteriolateral bundle in hyperflexion (130 to 140 degrees) ,revision can be done using a new anatomical ,visualization in the notch is obscured when femoral tunnel and femoral end can be the knee is placed in hyperflexion, due to poor 
circulation of the arthroscopic inflow fluid and debris from drilling the femoral tunnel and dragging of the fat pad into the femoral notch ,technically more demanding, endobutton is resting on the cortex of cancellous bone and working with the knee in hyperflexion causes a loss of the normal anatomical relationships in the notch, leading to spatial disorientation. Advantages of the transtibial tunnel technique are that it is familiar to most surgeons, it is simple and quick and it does not require the knee to be flexed beyond $90^{\circ}$ of flexion when the femoral tunnel is drilled. The major disadvantage of the transtibial tunnel technique is that it is not possible to independently drill the ACL femoral tunnel. Anatomical and clinical studies have demonstrated that the transtibial tunnel technique tends to place the tibial tunnel too posterior and the femoral tunnel too high and deep in the intercondylar notch. The purpose of our study was to compare the stability and functional outcome achieved with each technique in nonathletic population.

\section{Materials and methods:}

50 patients (39males and 11 females),all non-athletes with ACL deficient knees underwent ACL reconstruction, 25 by transtibial and 25 by anatomicaltechnique. This was a randomized control trial. All patients were operated by a single surgeon. Patients from both the groups were evaluated on the basis on stability using Lachman's and Slocum's tests and functional outcome using Lysholm knee score at 3,6 and 12 months. The duration of study was 3 years. All surgeries were performed under spinal anesthesia under tourniquet control.Quadrupled hamstring graft (Semitendinosus and Gracilis) was used.Thetibial tunnel was prepared using a standard tibial angle guide. The femoral tunnel was drilled either through the tibial tunnel(Transtibial technique) or the anteromedial portal(anatomical technique). The tibial side of the graft was fixed using an interference screw and the femoral tunnel with either interference screw or endobutton. The graft was cycled 15 to 20 times before closure of portals. All patients underwent a same physiotherapy protocol.

\section{Results:}

Demographic data was comparable in both groups. The the mode of injury and injury to surgery interval was similar in both groups.the length of femoral tunnel was similar in both groups.there was no statistical difference in range of motion at 3,6 and 12 month. There was no significant difference in the Functional outcome (Lysholm Knee score), anteroposterior stability (Lachman's test) and rotational stability (Slocum's test) $\{\mathrm{p}$ values $>0.05\}$.

\section{Conclusion:}

From our study we conclude that-

1) Both groups have equally good stability in both the anteroposterior and rotational plane.

2) Both groups have a good functional outcome in non-athletic group of individuals.

\section{Key Words:}

transtibial tunnel, anatomical tunnel, non athlete, ACL injury

Conflict of Interest: Nil Source of Support: None

\section{Bibliography}

1. Aglietti P, Buzzi R, Menchetti PM, Giron F. Arthroscopically assisted semitendinosus and gracilis tendon graft in reconstruction for acute anterior cruciate ligament injuries in athletes. Am J Sports Med 1996; 24: 726-31.

2. Arnold MP, Kooloos J, van Kampen A (2001) Single-incision technique misses the anatomical femoral anterior cruciate ligament insertion: a cadaver study. Knee Surg Sports TraumatolArthrosc 9:194-199

3. Bedi A, Altchek DW; The "footprint" anterior cruciate ligament technique: an anatomic approach to anterior cruciate ligament reconstruction.Arthroscopy. 2009 Oct;25(10):1128-38.

4. Gonzalo Samitier, Pedro Álvarez; Anteromedial portal versus transtibial drilling techniques in ACL reconstruction: a blinded cross-sectional study at two- to five-year follow-up; INTERNATIONAL ORTHOPAEDICS; Volume 34, Number 5 (2010), 747-754.

5. Behrendt S, Richter J. Anterior cruciate ligament reconstruction: drilling a femoral posterolateral tunnel cannot be accomplished using an over-the-top step-off drill guide. Knee Surg Sports TraumatolArthrosc. 2010; 18(9):12521256.

6. Brophy RH, Pearle AD. Single-bundle anterior cruciate ligament reconstruction: a comparison of conventional, central, and horizontal singlebundle virtual graft positions. Am J Sports Med. 2009; 37(7):1317-1323.

7. Jepsen CF, Lundberg-Jensen AK, Faunoe P; Does the position of the femoral tunnel affect the laxity or clinical outcome of the anterior cruciate ligament reconstructed knee? A clinical, prospective, randomized, double-blind study. Arthroscopy. 2007 Dec;23(12):1326-33.

8. Pearle AD, Shannon FJ, Granchi C, Wickiewicz TL, Warren RF; Comparison of 3-dimensional obliquity and anisometric characteristics of anterior cruciate ligament graft positions using surgical navigation; Am JSports Med. 2008 Aug;36(8):1534-41.

9. Omer A.Ilahi, N. JanetVentura, Amad A.Qadeer; Femoral Tunnel Length: Accessory Anteromedial Portal Drilling Versus Transtibial Drilling; Arthroscopy. 2012 Apr; 28(4):486-91.

10. Arnold MP, Kooloos J, van Kampen A (2001) Single-incision technique misses the anatomical femoral anterior cruciate ligament insertion: a cadaver study. Knee Surg Sports Traumatol Arthrosc 9:194-199.

11. Pearle AD, Shannon FJ, Granchi C, Wickiewicz TL, Warren RF; Comparison of 3-dimensional obliquity and anisometric characteristics of anterior cruciate ligament graft positions using surgical navigation; Am JSports Med. 2008 Aug;36(8):1534-41.

12. Behrendt S, Richter J. Anterior cruciate ligament reconstruction: drilling a femoral posterolateral tunnel cannot be accomplished using an over-the-top step-off drill guide. Knee Surg Sports TraumatolArthrosc. 2010; 18(9):12521256.
How to Cite this Article:

Electricwala A, Latkar C, Patil S, Jog V, Mahajan A, Deshpande S. Transtibial vs Anatomical tunneling techniques for arthroscopic ACL Reconstruction in non-athletic population. Journal Medical Thesis 2013 July-Sep; 1(1):35-36 